\title{
Correction to: Latino Identity and Political Attitudes
}

Correction to:

A. Saavedra Cisneros, Latino Identity and Political Attitudes, https://doi.org/10.1007/978-3-319-33969-6

The original version of this book was published with the family name of the author as 'Cisneros' in all the chapters. This has been now updated as 'Saavedra Cisneros' for all the chapters.

The updated original online version of the book can be found at https://doi.org/10.1007/978-3-319-33969-6

(C) The Author(s) 2019

A. Saavedra Cisneros, Latino Identity and Political Attitudes, https://doi.org/10.1007/978-3-319-33969-6_10 